\title{
Effectiveness of 8- or 12-weeks of ledipasvir and sofosbuvir in real-world treatment-naïve, genotype 1 hepatitis $C$ infected patients
}

\author{
M. P. Curry ${ }^{1}$ \\ E. B. Tapper $^{2}$ \\ B. Bacon $^{3}$ \\ D. Dieterich ${ }^{4}$ \\ S. L. Flamm ${ }^{5}$ \\ L. Guest ${ }^{6}$ \\ K. V. Kowdley ${ }^{7}$ \\ Y. Lee 6 \\ S. Milligan ${ }^{6}$ \\ N. Tsai $^{8}$ \\ Z. Younossi ${ }^{9}$
}

N. H. Afdhal ${ }^{1}$

${ }^{1}$ Division of Gastroenterology and Hepatology, Department of Medicine, Beth Israel Deaconess Medical Center, Harvard Medical School, Boston, MA, USA

${ }^{2}$ Division of Gastroenterology and Hepatology, University of Michigan, Ann Arbor, MI, USA

${ }^{3}$ Division of Gastroenterology/ Hepatology, Saint Louis University School of Medicine, Saint Louis, MO, USA

${ }^{4}$ Division of Liver Diseases, Department of Medicine, Mount Sinai School of Medicine, New York, NY, USA

${ }^{5}$ Division of Hepatology, Northwestern University Feinberg School of Medicine, Chicago, IL, USA

${ }^{6}$ Trio Health Analytics, La Jolla, CA, USA

${ }^{7}$ Liver Care Network, Swedish Medical Center, Seattle, WA, USA

${ }^{8}$ The Liver Center, Queen's Medical Center, Honolulu, HI, USA

${ }^{9}$ Center for Liver Diseases, Department of Medicine, Inova Fairfax Hospital, Falls Church, VA, USA

\section{Correspondence}

Dr. MP Curry, 110 Francis Street, Suite 8E, Boston, MA, USA.

Email: mcurry@bidmc.harvard.edu

Funding information

Gilead Science Inc.

\section{Summary}

Background: Treatment of genotype 1 hepatitis $\mathrm{C}$ virus (HCV) infection with combination direct acting anti-virals is associated with very high rates of sustained virological response (SVR). Daily combination of ledipasvir and sofosbuvir for 12 weeks is approved for the treatment of genotype $1 \mathrm{HCV}$ patients, though noncirrhotic patients who are naive to treatment with a baseline HCV RNA $<6$ million $\mathrm{IU} / \mathrm{mL}$ can be treated for 8 weeks. This guidance stemmed from a post hoc analysis of the ION 3 clinical trial, which demonstrated similar SVR for patients treated with ledipasvir and sofosbuvir with or without ribavirin for 8 or 12 weeks.

Aim: To compare the SVR for 8 weeks vs 12 weeks of ledipasvir and sofosbuvir in HCV infected patients in a real-world setting.

Methods: We performed an observational real-world cohort study of treatment success following 8 or 12 weeks of ledipasvir and sofosbuvir for treatment-naïve genotype $1 \mathrm{HCV}$ patients.

Results: A total of 826 patients were treated for either $8(n=252)$ or 12 weeks ( $n=574$ ) with ledipasvir and sofosbuvir and achieved SVR rate of $95.3 \%$ and there was no statistical difference in SVR rates in the two groups irrespective of any clinical or virological variables.

Conclusions: In treatment-naïve HCV genotype 1 patients, SVR was $95 \%$ in those treated for either 8 weeks or 12 weeks with ledipasvir and sofosbuvir. 8 week ledipasvir and sofosbuvir can reduce costs without compromising outcomes for those patients who qualify for such regimen. 


\section{1 | INTRODUCTION}

\section{1 | Background}

There is an estimated $80-185$ million people infected with Hepatitis $\mathrm{C}$ virus (HCV) worldwide. ${ }^{1,2}$ Despite the reduction in new infections in recent years, morbidity and mortality related to chronic infection are likely to increase. ${ }^{3}$ Since 2011 , there has been the development of several new regimens of direct acting anti-virals associated with significant improvements in efficacy and tolerability in treatment of $\mathrm{HCV}$. Eradication of HCV is associated with decreased overall morbidity and mortality as well as increased quality of life and reduced healthcare utilisation. ${ }^{4,5}$ Based on the prevalence of infection and availability of highly effective direct acting antivirals, treatment is now recommended for all patients with chronic HCV infection.

However, due to the high wholesale cost of direct acting antiviral treatment regimens, one of the commonly cited barriers to treatment is the cost of therapy. ${ }^{6}$ Despite this high cost, treatment of naïve genotype $1 \mathrm{HCV}$ patients is considered to be a cost-effective strategy when compared with other accepted medical practices. $^{7-9}$ Ledipasvir and sofosbuvir fixed dose combination (LDV/ SOF) is approved by the Food and Drug Administration for the treatment of genotype $1 \mathrm{HCV}$ infection in treatment-naïve patients with and without cirrhosis based on two registration trials called ION-1 and $10 N-3 .^{10,11}$ The $10 N-3$ study of treatment-naive noncirrhotic patients investigated LDV/SOF with or without ribavirin for 8 or 12 weeks and LDV/SOF for 12 weeks. Treatment-naïve noncirrhotic patients infected with genotype 1 who received LDV/SOF for 8 weeks achieved an SVR rate of $94 \%$. This SVR was not inferior to a 12-week regimen of LDV/SOF in an intent-to-treat analysis. Relapse rates were found to be higher in the cohort of patients randomised to 8 weeks of treatment regardless of ribavirin. However, in a post hoc analysis of the ribavirin-free treatment arms, patients with baseline HCV RNA levels $<6$ million $\mathrm{IU} / \mathrm{mL}$ were found to have similarly high SVR rates and low relapse rates regardless of 8- or 12week treatment durations. While this analysis was not controlled, the Food and Drug Administration included consideration of 8 weeks of LDV/SOF in genotype 1 treatment-naïve patients without cirrhosis who have a pre-treatment viral load of $<6$ million IU/ $\mathrm{mL} .^{12}$ Despite the approval of the Food and Drug Administration, the American Association for Study of Liver Disease/Infectious Diseases Society of America guidelines for the treatment of HCV state that shortening of therapy for less than 12 weeks is not recommended in African-American patients, patients with Human Immunodeficiency Virus infection and patients with known interleukin-28B polymorphism CT or TT. ${ }^{13}$ Guidelines from the European Association for the Study of the Liver state that treatment may be shortened to 8 weeks in treatment-naïve patients without cirrhosis if their baseline HCV RNA level is below 6 million $(6.8 \mathrm{Log}) \mathrm{IU} / \mathrm{mL}$, however, caution should be exercised especially in patients with F3 fibrosis, pending confirmation of these results in real-life studies. ${ }^{14}$ Last, the English National Health Service guidance recommends 8 weeks of LDV/SOF for noncirrhotic treatment-naïve genotype $1 \mathrm{HCV}$ infected patients regardless of HCV viral load. ${ }^{15}$ Several heterogeneous realworld cohort studies have reported excellent SVR rates for patients treated for 8 weeks with LDV/SOF \pm ribavirin. ${ }^{16-23}$

The importance of these recommendations for the possible shortening of duration cannot be underestimated as it is likely that there is a significant population of patients who meet these criteria who could benefit from a shortened, and thus less costly, course of treatment. However, the baseline HCV viral load cut off of 6 million $\mathrm{IU} / \mathrm{mL}$ has been called into question, as the post hoc analysis was underpowered and may reflect a statistical artefact. ${ }^{24}$

\subsection{Aim}

We chose to evaluate SVR rates following LDV/SOF without ribavirin for genotype 1, treatment-naïve patients in a real-world cohort of patients who have been treated for 8 or 12 weeks.

\section{PATIENT AND METHODS}

\subsection{Study design/setting}

The study cohort comprises treatment-naive, genotype 1 patients treated in community and academic practices affiliated with the Trio Health's Innovation Platform. Patients received LDV/SOF for either 8 or 12 weeks at the discretion of their treating physician. Data were collected through Trio Health's Innovation Platform, a unique platform that has the ability to collect and aggregate real-time data for disease management. Baseline information as well as outcomes data were collected through both the specialty pharmacies and clinicians in academic and community practices that work with Trio Health and were entered into the portal via a combination of nightly file feeds and manual user entry. In addition to data collection, the portal also scrubbed and mapped data, applied proprietary logic to identify errors, and prompted specialty pharmacies and clinicians to input data to ensure all data were complete and accurate. For this study, de-identified data were collected from this process, which was approved as an Institutional Review Board exemption under category 45 CFR 46.

This retrospective review of a prospectively defined cohort is based on data from treatment-naïve patients who were treated for 8 or 12 weeks with LDV/SOF between October 2014 and March 2015. Patients were recruited in real-world community and academic practices affiliated with Trio Healthcare. All patients were treatment naïve and received LDV/SOF without ribavirin for 8 or 12 weeks. The primary endpoint was SVR12, defined by a negative hepatitis C viral load by polymerase chain reaction assessed at 12 weeks following the completion of therapy. Treatment start and end dates were defined by pharmacy dispensing records. Additional clinical characteristics were recorded, including age, gender, ethnicity, physicianreported fibrosis burden, transplant status, comorbidities (human immunodeficiency virus [HIV], hepatitis B [HBV], diabetes), treatment duration and physician practice type. Collected laboratory values include HCV genotype, initial viral load, alanine and aspartate 
aminotransferase (ALT, AST), haemoglobin, and platelet count. Assessment of HCV RNA was performed as per standard clinic practice by the treating physician and reported in standardised international units. Fibrosis stage was determined by liver biopsy, serum biomarkers and vibration controlled elastography assessment by FibroScan as per the usual practice and was reported by the treating physician. Fibrosis staging was not performed in $12.6 \%$ of the patients enrolled,

\section{2 | Statistical analysis}

Associations between each individual potential predictor variable and the SVR modelled both as a "per protocol" and "intention to treat" were assessed. Predictor variables were entered into univariable ordinal regression models with fibrosis stage as the ordinal outcome. Predictor variables with two-tailed $P \leq .25$ were entered into multivariable logistic regression models. Manual backward stepwise elimination was used to generate the best-fitting multivariable logistic model. Forced adjustments for severe fibrosis and thrombocytopenia were subsequently performed for each variable in logistic regression analyses. These analyses were repeated for the overall cohort in addition to subgroup analyses for patients treated for 8 and 12 weeks.

\section{3 | RESULTS}

\section{1 | Treatment cohort}

A total of 826 patients received LDV/SOF treatment for either 8 or 12 weeks. Two hundred and fifty two patients were treated for 8 weeks and 574 were treated for 12 weeks (Figure 1). Fifty-one per cent of patients were male, $52 \%$ were Caucasian and $17 \%$ of patients were African American. All patients were treatment-naïve. The majority (63\%) of patients were treated in community practices and there was no difference in the duration of therapy between community and academic practices. Baseline characteristics of the cohort are presented in Table 1. Patients receiving 12 weeks of treatment were significantly older, more likely to be male gender, have more advanced fibrosis, have lower platelet counts, and have higher viral loads.

There were 587 who were deemed not to have liver cirrhosis by liver biopsy, FibroScan or serum fibrosis markers, and had a platelet count of $>100000 / \mu \mathrm{L}$ and an AST to platelet index (APRI) of $<1.0$. Two hundred and thirty-nine patients did not have formal staging of liver fibrosis or had a platelet count of $<100000 / \mu \mathrm{L}$ or an APRI of $>1.0$. Sustained virological response rates were assessed for the entire cohort of patients $(n=826)$ and for those formally deemed not to have liver cirrhosis.

\section{2 | Virological outcomes}

SVR12 was achieved in $787 / 826(95.3 \%)$ of patients in the entire cohort. Twenty-one patients were lost to follow-up, seven patients

\section{Treatment-naïve, non- cirrhotic $(n=826)$}

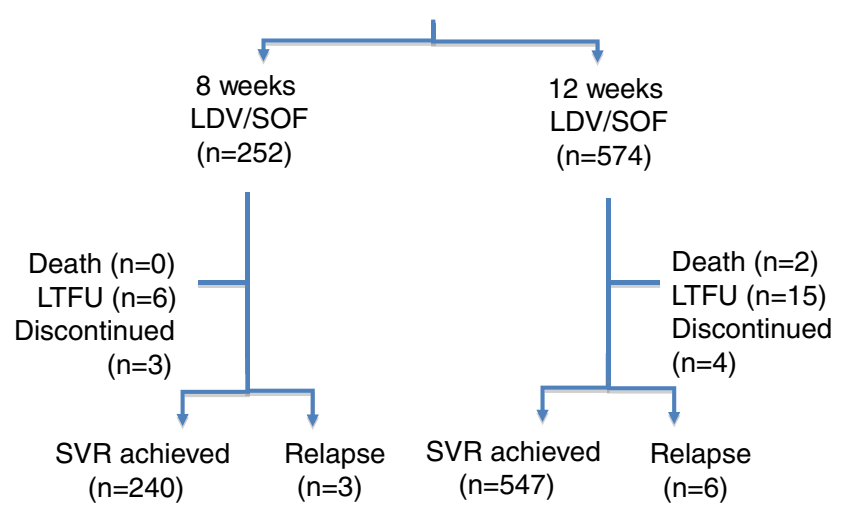

FIGURE 1 Distribution of all patients who were treated with LDV/SOF for 8 and 12 weeks. The number of patient achieving SVR and those experiencing relapse are shown for 8 and 12 weeks of treatment. The numbers of patient who died, discontinued therapy or who were lost to follow-up (LTFU) are displayed for both treatment durations

TABLE 1 Baseline characteristics of patients

\begin{tabular}{|c|c|c|c|}
\hline & $\begin{array}{l}8 \text { weeks } \\
(\mathrm{n}=252)\end{array}$ & $\begin{array}{l}12 \text { weeks } \\
(n=574)\end{array}$ & $P$ value \\
\hline Age >65 years, $n(\%)$ & $65(25.8)$ & $188(32.8)$ & .05 \\
\hline Male, n (\%) & $114(45.2)$ & $310(54.0)$ & .02 \\
\hline \multicolumn{4}{|l|}{ Race, n (\%) } \\
\hline Black & $37(15.9)$ & $106(19.8)$ & .07 \\
\hline Hispanic/Latin & $14(6.0)$ & $31(5.8)$ & \\
\hline White & $145(62.2)$ & $282(52.7)$ & \\
\hline Genotype 1a, n (\%) & $172(69.4)$ & $380(66.9)$ & .86 \\
\hline $\begin{array}{l}\text { Initial viral load, IU/mL } \\
\text { median }\left(10^{6}\right)(\mathrm{IQR})\end{array}$ & $1.33(0.39-2.33)$ & $2.56(0 / 89-6.23)$ & .0001 \\
\hline Academic practice, $\mathrm{n}(\%)$ & $83(32.9)$ & $219(38.2)$ & .16 \\
\hline Advanced fibrosis, $\mathrm{n}(\%)$ & 32 (12.7) & $142(24.7)$ & .0001 \\
\hline $\begin{array}{l}\text { Platelets }<100 \mathrm{~K} / \mathrm{mL} \text {, } \\
\mathrm{n}(\%)\end{array}$ & $2(0.79)$ & $21(3.7)$ & .02 \\
\hline Post-transplant, N (\%) & $1(0.4)$ & $4(0.7)$ & .60 \\
\hline Diabetes & $24(9.8)$ & 75 (13.3) & .04 \\
\hline
\end{tabular}

discontinued therapy and there were two deaths during treatment. None of the treatment discontinuations were related to study medications. Patients lost to follow-up or those that discontinued treatment are considered treatment failures in the intent-to-treat analysis. Intent-to-treat SVR12 was $95.2 \%$ for patients treated for 8 weeks and $95.3 \%$ for patients treated for 12 weeks. There was no difference in SVR by viral subtype regardless of duration of treatment (Figure 2). There were nine relapses in total, with three patients treated for 8 weeks for a relapse rate of $1.2 \%$. Six patients in the 12 -weeks treatment group relapsed for a relapse rate of $1.1 \%$ (Figure 1 and Table 2). Five hundred and seventy-seven patients met the $\mathrm{HCV}$ viral load of $<6$ million $\mathrm{IU} / \mathrm{mL}$ to receive 8 weeks of LDV/ 

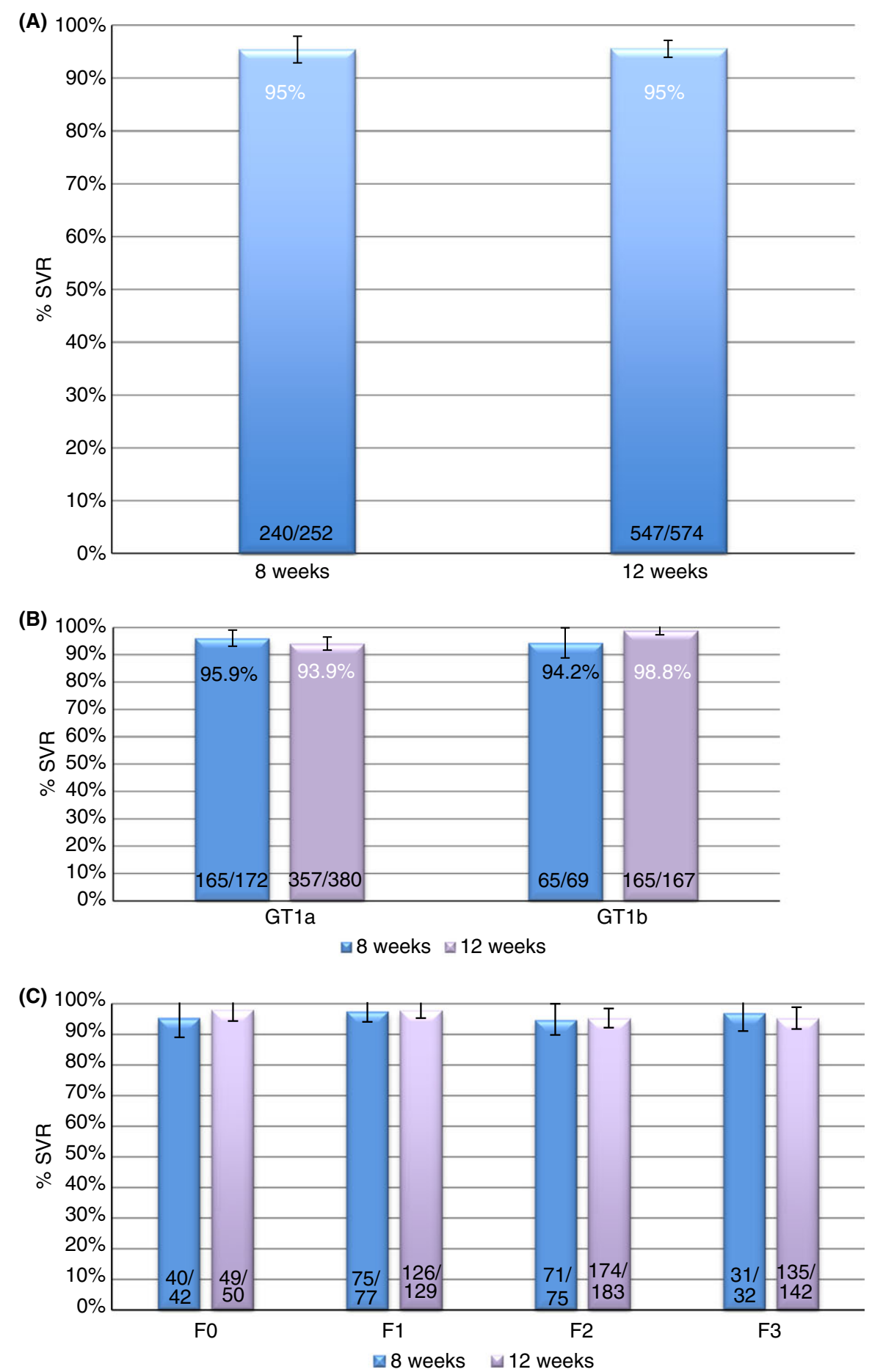

FIGURE 2 Overall SVR rates for all patients treated with LDV/SOF for 8 weeks (95\%) and 12 weeks (95\%) (A); SVR rates for patients with known genotype 1a vs $1 \mathrm{~b}$ for patients treated for 8 weeks (blue) and 12 weeks (purple) (B); SVR rates patients with known fibrosis scores receiving 8 weeks (blue) vs 12 weeks (purple) (C)

SOF. Two hundred and fifty-one patients (38\%) who were received 8 weeks of treatment achieved and SVR of $95.2 \%$, while $416(62 \%)$ patients with HCV RNA levels $<6$ million IU/mL and were eligible for 8 weeks but were treated for 12 weeks, had a similar SVR of $94.9 \%$.

Excluding patients without a formal stage of liver fibrosis, a platelet count of $<100000 / \mathrm{mL}$ and an APRI of $>1.0$, the SVR rate was $97 \%$ and was not different in those treated for 8 weeks (97\%) and those treated for 12 weeks (97\%). In this cohort, SVR was similar across all stage of liver fibrosis (FO-3) for 8 and 12 weeks (Figure 3). There was no difference in SVR noted in those who had diabetes mellitus vs those that did not with SVR rates of $95 \%$ seen in both cohorts. Per protocol analysis demonstrated that SVR rate in diabetics treated for 8 weeks was $96 \%$ and $98 \%$ in those treated for 12 weeks. There were 143 (17\%) African Americans included in the 
TABLE 2 Characteristics of all relapses

\begin{tabular}{|c|c|c|c|c|c|c|c|}
\hline Practice type & Patient age & Patient gender & Patient ethnicity (group) & Genotype group & Actual regimen name & Duration & Baseline VL \\
\hline Academic & 64 & $\mathrm{~F}$ & Black & $1 \mathrm{~A}$ & LDV-SOF & 8 & 805000 \\
\hline Academic & 67 & M & White & $1 \mathrm{~A}$ & LDV-SOF & 8 & 646400 \\
\hline Community & 61 & $\mathrm{~F}$ & White & $1 \mathrm{~A}$ & LDV-SOF & 12 & 3020000 \\
\hline Community & 61 & M & Black & $1 \mathrm{~A}$ & LDV-SOF & 12 & 3569042 \\
\hline Community & 58 & M & Black & 1UNKNOWN & LDV-SOF & 12 & 2245736 \\
\hline Academic & 46 & M & Unknown & $1 \mathrm{~A}$ & LDV-SOF & 12 & 2456117 \\
\hline Community & 63 & $\mathrm{~F}$ & Black & $1 \mathrm{~B}$ & LDV-SOF & 12 & 2977629 \\
\hline
\end{tabular}

study. SVR was achieved in $96 \%$ of African American patients treated with LDV/SOF. There were nine relapses in the entire cohort and six relapses occurred in African American patients. While the SVR rate in the patients treated for 8 weeks $(n=37)$ was numerically lower than those treated for 12 weeks $(n=106)$, this did not reach statistical significance. ( $89 \%$ vs $94 \% ; P=.29$ ). Again while there was no statistically significant difference in SVR rate noted between African American patients treated for 8 weeks and those African American patients who eligible to received for 8 weeks but were treated for 12 weeks ( $89 \%$ vs $95 \%$; $P=.28$ ), the result was numerically lower.

We examined the effect of baseline predictors on virological outcome and we were unable to identify any variable that was significantly associated with treatment failure in either per-protocol or intention-to-treat analyses in multivariable logistic regression models.

\section{DISCUSSION}

The efficacy and better tolerability of all oral anti-viral regimens in patients with chronic HCV infection is well established with SVR rates 21 -fold higher now than in the interferon era. ${ }^{25}$ These improvements in SVR are noted across multiple patient populations leading to the widespread use of these agents even in patients with advanced disease. ${ }^{26-28}$ However, due to the high cost of these regimens and the implementation of a triage system by health care agencies to allocate treatment, there are still significant populations of patients who have not yet been treated and are at risk of disease progression. Socioeconomic reasons have now replaced medical contraindications as the primary reason for patients to be denied access to treatment. ${ }^{29,30}$ Shortening the duration of treatment could significantly reduce the costs of treatment and in many cases, can be cost-saving. Post hoc analysis of the ION-3 registration trial identified a cohort of treatment-naive noncirrhotic patients with low viral load who are likely to have similar SVR rates to patients who received a 12-week treatment course of LDV/SOF. In view of this the Food and Drug Administration included the option for an 8-week duration of treatment for patients meeting these pretreatment criteria. International guidelines have suggested caution in the use of treatment regimens of less than 12 weeks in this population pending the results of real-world studies addressing shorter durations of treatment. Reducing the duration of treatment for these patients could result in significant cost savings and possibly allow more patients to receive much needed anti-viral therapy.

In this real-world experience of HCV treatment in genotype1 treatment-naïve patients, high SVR rates, comparable to that was observed in registration trials, were achieved. There were no differences in SVR between the 8- and 12-week cohorts and no differences were observed in SVR across genotype subtype or fibrosis stage. This analysis of a real-world cohort of patients treated in community and academic medical centres included some patients who did not have a formal assessment of liver fibrosis and had platelet counts of $<100000 / \mu \mathrm{L}$ or an APRI of $>1.0$. When we excluded these patients from the SVR analysis, the SVR rates were $97 \%$.

Five hundred and seventy-seven patients met the HCV viral load of $<6$ million $\mathrm{IU} / \mathrm{mL}$ criteria to receive 8 weeks of LDV/SOF. Two hundred and fifty-one patients (38\%) received 8 weeks of treatment and SVR was achieved in $95.2 \%$ while 416 (62\%) patients with HCV RNA levels $<6$ million $\mathrm{IU} / \mathrm{mL}$ were treated for 12 weeks and SVR was achieved in $94.9 \%$. Shortening the course of treatment by 4 weeks for these 416 patients could possibly have resulted in significant cost savings across the entire cohort.

Our data are similar to that recently published by loannou and colleagues who analysed the real-world effectiveness of DAA treatment in the Veterans Affairs National Health Care System. Twentyseven per cent of patients receiving LDV/SOF were treated for 8 weeks and achieved SVR rates of $94.3 \% .^{31}$ In a subgroup analysis of those treatment-naïve noncirrhotic patients with HCV RNA levels $<6$ million IU/mL, $48 \%$ were treated for 8 weeks and achieved SVR of $95 \%$, similar to the SVR achieved by the $38 \%$ of patients in our study treated for 8 weeks.

Prior studies have shown higher rates of virological relapse in genotype 1a patients after 8 weeks of treatment. In this real-world cohort, very low and no difference was observed in relapse rates across genotype subtype and treatment duration.

Our study is limited by the retrospective nonrandomised design that precludes rigid comparisons of the treatment duration. In addition, treatment duration was at the discretion of the treating 

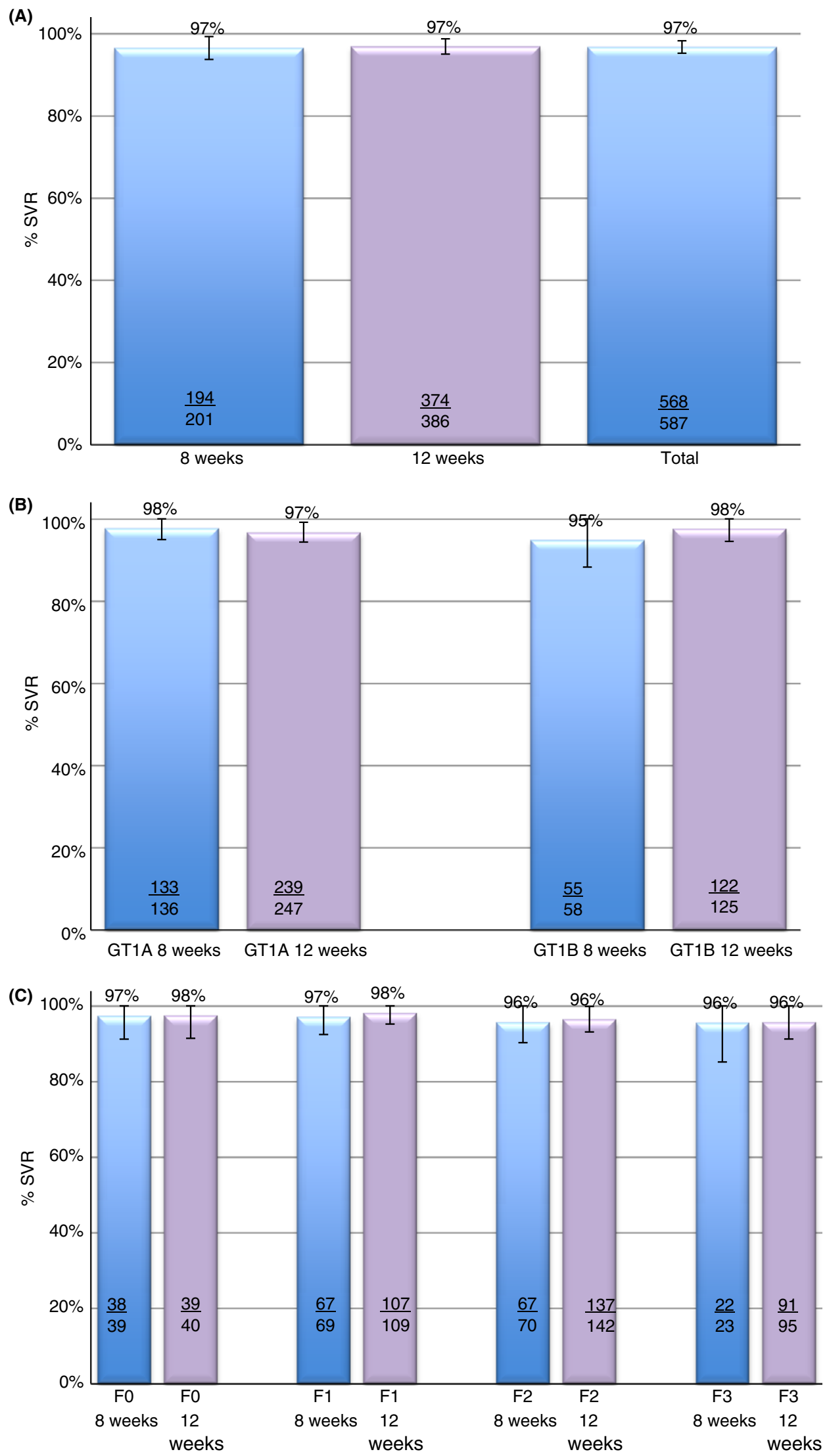

FIGURE 3 Overall SVR rates for patients without cirrhosis treated with LDV/SOF for 8 and 12 weeks (A); SVR rates for patients without cirrhosis with known genotype 1a vereses $1 \mathrm{~b}$ receiving 8 weeks (blue) and 12 weeks (purple) (B); and SVR rates for patient with known fibrosis scores receiving 8 weeks (blue) and 12 weeks (purple) (C) 
physician and as such there were significantly more patients older than 65 years, of male gender, diabetics and more advanced fibrosis patients in the cohort treated for 12 weeks. Details such as IL28B polymorphism were not available, however this assay is rarely used in the current era of direct acting anti-viral therapy. Additionally we do not have data on concomitant use of medications such as acidreducing medications for this study, though this is unlikely to have changed the results. ${ }^{32}$ Our entire cohort included some patients who did not have a formal assessment of liver fibrosis as well as patients who had platelet count of $<100000 / \mu \mathrm{L}$. To more stringently evaluate the efficacy of LDV/SOF for 8 vs 12 weeks in true non-cirrhotic patients, we further analysed the SVR rates in a cohort with formal assessment of liver fibrosis, platelet count of $>100000 / \mu \mathrm{L}$ and an APRI of $>1.0$ and showed excellent SVR rates of $97 \%$ in the 8 and 12 weeks cohorts (Figure 3).

Further limitations include the small numbers of patients for subgroup anlaysis, thus limiting the power of this study to determine significant differences in SVR by treatment duration in these subgroups. The overall SVR rate for African Americans treated with LDV/SOF was $96 \%$. The numbers of African American patients treated for 8 weeks in this study is small and though the SVR rates are not significantly different for 8 weeks and 12 weeks of treatment, there is a numerically lower SVR rate and relapse is more commonly seen in African American patients in this study (Table 2). The small numbers of African American patients in this study limits any definitive conclusions regarding 8 vs 12 weeks in this population. As with other studies of African American patients, which demonstrate similar SVR rates for 8 and 12 weeks we suggest that caution be used in shortening treatment in this population because of a higher relapse rate. $^{33,34}$

Despite these limitations, our study has comparable rates of SVR to that observed in registration trials for LDV/SOV. ${ }^{10}$ Our study also supports the real-world effectiveness study by loannou and colleagues for the VA cooperative and supports the VA treatment guidelines and the English National Health Service guidance on shortening duration of treatment in noncirrhotic treatment-naïve patients with HCV RNA $<6$ million IU/mL. ${ }^{31,35}$ The generalisability of the clinical data from ION-3 has also been demonstrated by multiple other, real-world studies comparing 8 vs 12 weeks of LDV/SOF multiple geographies and patient care settings including patients with HIV disease. ${ }^{17,19,20,22,36-38}$

In conclusion, our study supports the FDA recommendation that clinicians should consider 8 weeks of LDV/SOF for initial treatment of HCV genotype 1, treatment-naïve, non-cirrhotic patients.

\section{ACKNOWLEDGEMENTS}

Declaration of personal interests: MP Curry reports receiving research support from Gilead Sciences Inc. and Conatus, and consulting, advisory board from Gilead Sciences Inc., Bristol Myers Squibb, AbbVie and Trio Health; EB Tapper has no conflict of interest; B Bacon reports receiving research support from Merck, Gilead Sciences Inc. Bristol Myers Squibb, and AbbVie, and consulting/speakers bureau and advisory board/data safety monitoring board membership from Merck, Gilead Sciences Inc., Bristol Myers Squibb, ISIS Pharmaceuticals, AbbVie, Valeant Pharmaceuticals, Trio Health and Janssen Pharmaceuticals; D Dieterich reports receiving grant support or consulting fees from Gilead Sciences, Bristol Myers Squibb, AbbVie, Merck, Trio Health and Janssen Pharmaceuticals; S Flamm reports receiving grant support form Gilead Sciences Inc, AbbVie, Bristol Myers Squibb and Merck and consulting fees from Gilead Sciences Inc, AbbVie, Bristol Myers Squibb, Merck, Trio Health and Janssen; $L$ Guest reports receiving grant support from Gilead Sciences and AbbVie and she is an employee of Trio Health; K Kowdley reports receiving grant support from AbbVie, Evidera, Galectin, Gilead Sciences, Immuron, Intercept Pharmaceuticals, Merck, NGM Biopharma, Novartis, Tobira Therapeutics and serves as a consultant or advisor to AbbVie, Enanta, Gilead Sciences, Intercept Pharmaceuticals, Merck, Trio Health and Verlyx; $Y$ Lee reports receiving grant support from Gilead Sciences and AbbVie and she is an employee of Trio Health; S Milligan reports receiving grant support from Gilead Sciences and AbbVie and she is an employee of Trio Health; N Tsai reports receiving advisory and speakers bureau fees from Gilead Sciences, Bristol Myers Squibb, AbbVie, Merck, Intercept Pharmaceuticals, Janssen Pharmaceuticals, Bayer, Trio Health and Valeant/Salix Pharmaceuticals and grant support from Gilead Sciences, Bristol Myers Squibb, AbbVie, Merck, Intercept Pharmaceuticals, Janssen Pharmaceuticals and Bayer and receives grant support from Gilead Sciences Inc, Bristol Myers Squibb, AbbVie, Merck, Intercept Pharmaceuticals, Janssen Pharmaceuticals and Bayer; Z Younossi, receiving consulting fees from Gilead Sciences, AbbVie, Intercept Pharmaceuticals, Bristol Myers Squibb, Trio Health and GlaxoSmithKlinePLC; $\mathrm{NH}$ Afdhal reports receiving grant support from Gilead Sciences Inc, AbbVie, and Bristol Myers Squibb and consulting/advisory board fees from Merck, Gilead Sciences Inc, Echosens, GlaxoSmithKline PLC, Ligand Pharmaceuticals, Inc, Janssen Pharmaceuticals Inc, Roivant Sciences Inc, Co-Crystal Pharma Inc, Trio Health and Shionogi, Inc and being employees of and having an equity interest in Springbank Pharmaceuticals, Allurion Technologies and Gilead Sciences Inc.

\section{AUTHORSHIP}

Guarantor of the article: Michael Curry.

Author contributions: Afdhal: concept; Curry, Tapper, Guest, Milligan, Lee, Afdhal: analysis; Afdhal, Bacon, Curry, Dieterich, Flamm, Kowdley, Tsai, Younossi: data acquisition; Curry: writing; Afdhal, Bacon, Curry, Dieterich, Flamm, Guest, Kowdley, Lee, Milligan, Tsai, Younossi: criticial revisions.

All authors approved the final version of the manuscript.

\section{REFERENCES}

1. Mohd Hanafiah K, Groeger J, Flaxman AD, Wiersma ST. Global epidemiology of hepatitis $C$ virus infection: new estimates of age-specific antibody to HCV seroprevalence. Hepatology. 2013;57:13331342. 
2. Gower E, Estes C, Blach S, Razavi-Shearer K, Razavi H. Global epidemiology and genotype distribution of the hepatitis $C$ virus infection. J Hepatol. 2014;61(Suppl 1):S45-S57.

3. Razavi H, Waked I, Sarrazin C, et al. The present and future disease burden of hepatitis C virus (HCV) infection with today's treatment paradigm. J Viral Hepat. 2014;21(Suppl 1):34-59.

4. van der Meer AJ, Veldt BJ, Feld JJ, et al. Association between sustained virological response and all-cause mortality among patients with chronic hepatitis $\mathrm{C}$ and advanced hepatic fibrosis. JAMA. 2012;308:2584-2593.

5. Younossi ZM, Stepanova M, Sulkowski M, Naggie S, Henry L, Hunt S. Sofosbuvir and ledipasvir improve patient-reported outcomes in patients co-infected with hepatitis $\mathrm{C}$ and human immunodeficiency virus. J Viral Hepat. 2016;23:857-865.

6. Iyengar S, Tay-Teo K, Vogler S, et al. Prices, costs, and affordability of new medicines for hepatitis $C$ in 30 countries: an economic analysis. PLoS Med. 2016;13:e1002032.

7. Chhatwal J, He T, Lopez-Olivo MA. Systematic review of modelling approaches for the cost effectiveness of hepatitis $C$ treatment with direct-acting antivirals. Pharmacoeconomics. 2016;34:551-567.

8. Chhatwal J, Kanwal F, Roberts MS, Dunn MA. Cost-effectiveness and budget impact of hepatitis $C$ virus treatment with sofosbuvir and ledipasvir in the United States. Ann Intern Med. 2015;162: 397-406.

9. Younossi ZM, Park H, Saab S, Ahmed A, Dieterich D, Gordon SC. Cost-effectiveness of all-oral ledipasvir/sofosbuvir regimens in patients with chronic hepatitis $C$ virus genotype 1 infection. Aliment Pharmacol Ther. 2015;41:544-563.

10. Kowdley KV, Gordon SC, Reddy KR, et al. Ledipasvir and sofosbuvir for 8 or 12 weeks for chronic HCV without cirrhosis. N Engl J Med. 2014:370:1879-1888.

11. Afdhal N, Zeuzem S, Kwo $P$, et al. Ledipasvir and sofosbuvir for untreated HCV genotype 1 infection. N Engl J Med. 2014;370:18891898.

12. US Food Drug Administration. Centre for drug evaluation and research. Sofosbuvir and ledipasvir fixed-dose combination, in 2014: NDA 205834. https://www.accessdata.fda.gov/drugsatfda_docs/ nda/2014/205834Orig1s000MedR.pdf. Accessed January 2017.

13. AASLD-IDSA. Initial treatment fo HCV infection. http://www.hcvgui delines.org/full-report/initial-treatment-hcv-infection Accessed July 2016.

14. European Association for Study of Liver. EASL recommendation on treatment of heaptitis C 2015. J Hepatol. 2015;63:199-236.

15. UK National Institute for Health Care and Excellence. Appraisal consultation document. Ledipasvir-sofosbuvir for treating chronic hepatitis C, 2015. https://www.nice.org.uk/guidance/ta363. Accessed January 2017.

16. Marshall VJ, Rife K, Hirsch A, et al. Real life treatmet outcomes with 8 week course treatment for hepatitis $\mathrm{C}$ without cirrhosis confirmed by transient elastography. Hepatology. 2015;62(Suppl 1):779A

17. Roytman M, Tang AL, Wu C, et al. Real Life Experience with Sofosbuvir and Ledipasvir fixed does regemine in the multicentric cohort of patients with chronic hepatitis C. Hepatology. 2015;62(Suppl 1):761A.

18. Buggisch B, Boker K, Gunther R, et al. Ledipasvire/Sofosbuvir (LDV/ SOF) for 8 weeks in genotype 1 (GT1) treastment-naive non cirrhotic patients with $\mathrm{HCV}$ viraload $<6$ millionsIU/ML $(6 \mathrm{M})$ : a comparative analysis of the phase-3 ION-3 data and real world effectiveness. J Hepatol. 2016;64:S810.

19. Crespo J, Fernandez I, Cabezas J, et al. Effectiveness and safety of sofosbuvir / Ledipasvir treatment for monoinfected genotype $1 \mathrm{HCV}$ patients in rela life clinical practice: results fo the Spanish Hepa-C cohort. J Hepatol. 2016;64:S217.
20. Lai JB, Witt MA, Witt DJ. Real World Effectiveness of 8,12 and 24 weeks of Ledipasvire(LDV)/Sofosbuvir(SOF)- based therapy for heaptitis $\mathrm{C}(\mathrm{HCV})$ genotype 1: analysis in a large integrated health care system. J Hepatol. 2016;64:S778.

21. Latt NL, Gevorkyan R, Yanny BT, Sahota A. Ledipasvir / Sofosbuvir for 8 weeks in non cirrhotic treatment naive patients with genotype 1 hepatitis $C$ infection: real life experience in a community setting. J Hepatol. 2016;64:S802.

22. Qureshi K, Andres J, Regester J, et al. Real-Life outcomes of 8 weeks regimen of sofosbuvir and ledipasvir without ribavirin in non cirrhotics treatment naive hepatitis C genotype 1 patients with less thatn 6 millions IU/ML viral load. J Hepatol. 2016;64:S786.

23. Terrault NA, Zeuzem S, Di Bisceglie AM, et al. Effectiveness of ledipasvir-sofosbuvir combination in patients with hepatitis C Virus Infection and Factors Associated With Sustained Virologic Response. Gastroenterology. 2016;151:1131-1140 e5.

24. O'Brien TR, Feld JJ, Kottilil S, Pfeiffer RM. No scientific basis to restrict 8 weeks of treatment with ledipasvir/sofosbuvir to patients with hepatitis C virus RNA $<6,000,000 \mathrm{IU} / \mathrm{mL}$. Hepatology. 2016;63:28-30.

25. Moon AM, Green PK, Berry K, lannou GN. Transformation of hepatitis $C$ antiviral treatment in a national healthcare system following the introduction of direct antiviral agents. Aliment Pharmacol Ther. 2017;45:1201-1212.

26. Guarino M, Morisco F, Valvano MR, et al. Systematic review: interferon-free regimens for patients with HCV-related Child C cirrhosis. Aliment Pharmacol Ther. 2017;45:1193-1200.

27. Pillai AA, Maheshwari R, Vora R, et al. Treatment of HCV infection in liver transplant recipients with ledipasvir and sofosbuvir without ribavirin. Aliment Pharmacol Ther. 2017;45:1427-1432.

28. Reddy KR, Kim JK, Kuo A, et al. All-oral direct-acting antiviral therapy in HCV-advanced liver disease is effective in real-world practice: observations through HCV-TARGET database. Aliment Pharmacol Ther. 2017;45:115-126.

29. Dieterich D, Bacon BR, Curry MP, et al. Access to therapy in the era of all DAA regimens: real world experiences from the TRIO Network. Gastroenterology. 2016;150:S1040.

30. Younossi ZM, Bacon BR, Dieterich DT, et al. Disparate access to treatment regimens in chronic hepatitis $C$ patients: data from the TRIO network. J Viral Hepat. 2016;23:447-454.

31. Ioannou GN, Beste LA, Chang MF, et al. Effectiveness of Sofosbuvir, Ledipasvir/Sofosbuvir, or Paritaprevir/Ritonavir/Ombitasvir and Dasabuvir Regimens for Treatment of Patients With Hepatitis C in the Veterans Affairs National Health Care System. Gastroenterology. 2016; 151:457-471.

32. Tapper EB, Bacon BR, Curry MP, et al. Evaluation of proton pump inhibitor use on treatment outcomes with ledipasvir and sofosbuvir in a real-world cohort study. Hepatology. 2016;64:1893-1899.

33. Wilder JM, Jeffers LJ, Ravendhran N, et al. Safety and efficacy of ledipasvir-sofosbuvir in black patients with hepatitis $\mathrm{C}$ virus infection: a retrospective analysis of phase 3 data. Hepatology. 2016;63:437-444.

34. Backus LI, Belperio PS, Shahoumian TA, Loomis TP, Mole LA. Realworld effectiveness of ledipasvir/sofosbuvir in 4,365 treatmentnaive, genotype 1 hepatitis C-infected patients. Hepatology. 2016; 64:405-414.

35. Chronic hepatitis $\mathrm{C}$ virus (HCV) infection: treatment considerationsfrom the Department of Veternas Affairs National Hepatitis C Resource Center Program and national Viral Hepatitis Program. http://www.hepatitis. va.gov/provider/index.asp Accessed March 2017.

36. Terrault NA, Zeuzem S, Di Bisceglie AM, et al. Treatment Outcomes with 8,12 and 24 week regimens of Ledipasvir/Sofosbuvir for the treatment of heaptitis $C$ infection: analysis of a multicenter prospective, onservational study. Hepatology. 2015;62(Suppl 1):256A. 
37. Lai JB, Witt MA, Pauly MP, et al. Eight- or 12-week treatment of hepatitis C with ledipasvir/sofosbuvir: real-world experience in a large integrated health system. Drugs. 2017;77:313-318.

38. Ingiliz $\mathrm{P}$, Christensen $\mathrm{S}$, Kimhofer $\mathrm{T}$, et al. Sofosbuvir and ledipasvir for 8 weeks for the treatment of chronic hepatitis $C$ virus (HCV) infection in HCV-monoinfected and HIV-HCV-coinfected individuals: results from the German hepatitis C cohort (GECCO01). Clin Infect Dis. 2016;63:1320-1324.
How to cite this article: Curry MP, Tapper EB, Bacon B, et al. Effectiveness of 8- or 12-weeks of ledipasvir and sofosbuvir in real-world treatment-naïve, genotype 1 hepatitis $C$ infected patients. Aliment Pharmacol Ther. 2017;46:540-548.

https://doi.org/10.1111/apt.14204 